\title{
Inverse Taylor Series Problem in Linear Filtering and Related Conjectures
}

\author{
Xiao-Yun Lu MIEEE and Karl J. Hedrick \\ PATH, ITS, U. C. Berkeley
}

\begin{abstract}
This paper considers an inverse Taylor series problem and some related conjectures which arise in considering linear integral filters from a new viewpoint. The inverse Taylor series problem is defined as expanding a Lebesgue measurable function into a Taylor series by integration, which is an estimation problem.
\end{abstract}

\section{Introduction}

This note presents an inverse Taylor series problem and related mathematical conjectures which arises in the consideration of integral filters [3].

Suppose $\widehat{f}(t)$ is a measured signal which may be polluted by high frequency measurement noise. Mathematically, $f(t)$ may not be differentiable or even not continuous. The weakest possible mathematical assumption on $\hat{f}(t)$ is Lebesgue measurability. In engineering practice, one often needs to filter out noise from the signal $\widehat{f}(t)[4,5,6,1,2]$. For control application, beside filtering the measured signal, one also needs to estimate the derivative of the signal. It is well-known that integration not only can smooth up discontinuous functions, but also can filter out high frequency noise. This is the impetus for searching analog integral filters from a new viewpoint [3]. The main problem is how to build an integral filter which has enough information for the estimation or re-construction of the nominal signal and its derivative with higher accuracy. Although, classical analog linear integral filters have been extensively studied for the purpose of signal processing $[4,5,6]$, they are often frequency-selective type filters which are not quite fit for nonlinear control purposes. Kalman filters [1, 2] are for control purposes, but they need a model for the signal, which restricts its use in some cases.

Most recently, the authors found that one can construct integral filters from an inverse Taylor series approach. This problem has its own right in mathematics. The main idea is to build linear integral filters based on truncation of formal Taylor series and reconstruct required signal. Preliminary research in this respect has been presented in [3]. This paper concentrate on some related mathematical conjectures.

\section{Inverse Taylor Series Problem}

Suppose a real function $f(t)$ is Lebesgue integrable on real line $\mathbf{R}$. A neighborhood of a point is defined as

$$
N_{\delta}\left(x_{0}\right)=\left\{x|x \in \mathbf{R},| x-x_{0} \mid<\delta, \delta>0\right\}
$$

The following questions may be araised:

(1) Is it possible to approximately expand $f(t)$ as a Taylor series in $N_{\delta}\left(x_{0}\right)$ by integration?

(2) What is the error if one use information from such a Taylor development to estimate $f(t)$ itself and its derivatives?

To answer these questions leads to some new viewpoint on analog integral filters [3]. This short paper will present the inverse Taylor series problem and a related mathematical problem which is still open.

In general, the inverse Taylor series problem is proposed as follows.

In the well-known Taylor series development, if $f(t)$ is continuously differentiable $n+1$ times in $N_{\delta}\left(x_{0}\right)$ $\dot{f}(t), \ldots, f^{(n)}(t)$, unknown $f\left(t_{1}\right)$ is constructed when $t=t_{1}-\Delta t$ with $|\Delta t|$ sufficiently small. An important point here is that, to approximate $f\left(t_{1}\right)$ with $f(t)+f(t) \Delta t+\ldots+\frac{1}{n !} f^{(n)}(t)(\Delta t)^{n}$, the error is in the order of $O\left((\Delta t)^{n+1}\right)$.

Suppose that $f(t)$ is Lebesgue integrable with respect to $t$ and the value of $f(s)$ at an arbitrary time instant $s=t_{1}$ is known. Let $t=t_{1}-\Delta t$ with $|\Delta t|$ sufficiently small. It is to find the value of $f(s)$ at $s=t$ without resort to differentiation at time instant $t$. Construct a formal Taylor series as follows.

$$
\begin{gathered}
f\left(t_{1}\right)=f(t+\Delta t) \\
=f(t)+\dot{f}(t) \Delta t+\ldots+\frac{1}{n !} f^{(n)}(t)(\Delta t)^{n}+O\left((\Delta t)^{n+1}\right)
\end{gathered}
$$

where $f(t), \dot{f}(t), \ldots, f^{(n)}(t)$ are unknown and are to be found. Mathematically, this may be called an inverse Taylor series problem. 
To solve the inverse Taylor series problem, consider the following auxiliary dynamic system constructed from the formal Taylor series with the remainder $O\left((\Delta t)^{n+1}\right)$ dropped. Let $\tau=\Delta t$, and use $z_{i}(t)$ to replace $f^{(i-1)}(t), i=1, \ldots, n$. It is obtained that

$$
\begin{aligned}
\dot{z}_{1}= & z_{2} \\
\dot{z}_{2}= & z_{3} \\
& \cdots \ldots \\
\dot{z}_{n}= & \frac{n !}{\tau^{n}}\left(-z_{1}-\ldots-\frac{\tau^{i}}{i !} z_{i} \ldots-\frac{\tau^{n-1}}{(n-1) !} z_{n}+\widehat{f}(t)\right)
\end{aligned}
$$

where $\hat{f}(t)=f\left(t_{1}\right)$. In application, $\hat{f}(t)$ is a measured signal (in real-time or off-line).

Now one can estimate $f(t)$ which is a filtered counterpart of $\hat{f}(t)$ as well as the derivatives of $f(t)$ as follows. For some $T>0$, for $t \in[T, \infty)$

$$
\begin{aligned}
f_{-} f l t(t) & \approx z_{1}(t)+\ldots+\frac{\tau^{i}}{i !} z_{i+1}(t)+\ldots+\frac{\tau^{n-1}}{(n-1) !} z_{n} \\
\frac{d^{i}}{d t^{i}}(f(t)) & \approx z_{i+1}(t)
\end{aligned}
$$

This defines an integral filter which can be used in control and signal processing [3]. It should be noted that this is an asymptotic filter if the system (2.1) is globally asymptotically stable. As can be checked that it is true for $n=1, \ldots, 4$.

Different filter construction examples are refered to [3].

\section{Related Conjectures}

For the stability of linear differential equation (2.1) related to the inverse Taylor series problem, the following result is necessary.

Conjecture 1 For any integer $n$, the characteristic polynomial

$$
F(n, \tau, s)=\frac{n !}{\tau^{n}} s+\ldots+\frac{n !}{i ! \tau^{n-i}} s^{i}+\ldots+\frac{n !}{(n-1) ! \tau} s^{n-1}+s^{n}
$$

is Hurwitz for $\tau \in(0, r)$ or even $(0, \infty)$, where $r>0$. If it is not true, what is the largest integral $N$ such that $F(N, \tau, s)$ is Hurwitz?

Equivalently, if both side is multiplied by $\frac{\tau^{n}}{n !}$ and let $\lambda=\tau s$, it is obtained that

$$
G(n, \lambda)=1+\frac{1}{1 !} \lambda+\ldots+\frac{1}{i !} \lambda^{i}+\ldots+\frac{1}{n !} \lambda^{n} .
$$

Clearly, if $\lambda$ is a complex root of $G(n, \lambda)$, then $\frac{\lambda}{\tau}$ is a complex root of $F(n, \tau, s)$ for any $\tau>0$. This proves the following result.

Lemma $1 F(n, \tau, s)$ is Hurwitz for $\tau \in(0, \infty)$ if and only if $G(n, \lambda)$ is Hurwitz.
It is noted that $G(n, \lambda)$ does not depend on parameter $\tau$.

Equivalently, we have

Conjecture $2 G(n, \lambda)$ is Hurwitz for any positive integer $n$. If it is not true, what is the largest integer $N$ such that $G(N, \lambda)$ is Hurwitz?

Lemma 2 As a function of $\lambda, G(\infty, \lambda)$ has no finite complex root. i.e. $G(\infty, \lambda) \neq 0$ for any finite $\lambda \in \mathbb{Z}$ where $\mathbb{Z}$ is the complex plane.

Proof. Clearly,

$$
G(\infty, \lambda)=e^{\lambda}
$$

Let $\lambda=a+b j$ be complex number, where $a$ and $b$ are real numbers and $j^{2}=-1$.

$$
e^{\lambda}=e^{a}(\cos b+j \sin b)
$$

Then

$$
G(\infty, \lambda)=0 \Leftrightarrow \cos b+j \sin b=0
$$

which has no solution because

$$
\cos ^{2} b+\sin ^{2} b=1
$$

for any real number $b$. It is proved. $\diamond$

In fact, the only zero for $e^{\lambda}$ on the generalized complex plane is $\lambda=-\infty$. This leads to the following conjecture.

Conjecture 3 If $G(n, \lambda)$ has complex root $\lambda=\vartheta(n)$ for $n=1,2, \ldots$, Then

$$
\lim _{n \rightarrow \infty} \vartheta(n)=-\infty
$$

\section{References}

[1] R. E. Kalman, 1960, A new approach to linear filtering and prediction problems, Trans: ASME, J. Basic Eng., Vol. 82, p35-45

[2] R. E. Kalman and R. S. Bucy, New results in linear filtering and prediction theory, Trans. ASME, J. Basic Eng., Vol. 83, p95-108

[3] X. Y. Lu and K. J. Hedrick, 2000, Integral filters from a new viewpoint and their application in nonlinear control, Proc. IEEE Conference on Control Application, Sept. 25-27, Anchorage, Alaska

[4] A. V. Oppenheim, A. S. Willsky and S. H. Nawab, 1997, Signals and Systems, 2nd Ed., Prentice-Hall

[5] M. E. Van Valkenburg, 1982, Analog Filter Design, CBS College Publishing, New York

[6] R. E. Ziemer, W. H. Tranter and D. R. Fannin, 1989, Signals and Systems: Continuous and Discrete, 2ud Ed., Macmillan Publishing Company, New York 